\title{
Dificuldades apresentadas pelo modelo wiki para a implementação de um ambiente colaborativo de aprendizagem
}

\author{
Marcelo Augusto Rauh Schmitt \\ Escola Técnica - UFRGS
}

\begin{abstract}
Resumo
Este artigo apresenta uma análise dos problemas associados à utilização de wikis na aprendizagem colaborativa. Diversos autores concordam que há dificuldades para utilização do modelo wiki puro neste tipo de aplicação, o que também foi constatado na utilização do ambiente na UFRGS e será comentado no artigo. A busca de uma solução, com base no Modelo de Colaboração 3C, permitiu identificar que tais dificuldades estão associadas às atividades de coordenação e comunicação, que não têm suporte apropriado no ambiente wiki. Este trabalho propõe mecanismos explícitos de coordenação e comunicação para dirimir os problemas verificados e tornar o ambiente wiki mais adequado para a aprendizagem colaborativa.
\end{abstract}

\section{Introdução}

Os wikis são ambientes/ferramentas cada vez mais difundidos na Internet. Fazem parte do que está sendo denominado como web 2.0, ou seja, a segunda geração de ferramentas intermediadas pela web e voltadas ao apoio do trabalho colaborativo. Consistem em ambientes que permitem a construção coletiva de hipertextos de forma muito rápida e simplificada, não exigindo dos colaboradores conhecimento especializado de construção de páginas. Outra característica dos wikis é o dinamismo das páginas produzidas e a liberdade de alterar o que existe e acrescentar novas páginas. O principal exemplo do uso desse tipo de ambiente em uma escala muito significativa é a Wikipedia - enciclopédia virtual construída a partir da colaboração de qualquer voluntário. Apesar dos temores iniciais a respeito da suposta falta de confiabilidade das informações ali contidas, ela vem se constituindo em uma fonte de consulta respeitada e muito utilizada, competindo com enciclopédias tradicionais.

Em trabalho anteriormente publicado (Tarouco et alli, 2005), foi descrita uma experiência de uso de wiki como uma ferramenta de co-autoria em sala de aula evidenciando os mecanismos e modalidades de uso deste ambiente no processo de aprendizagem baseado na cooperação. O wiki é, sem dúvida, um ambiente que permite a cooperação e, especialmente, a cooperação não hierárquica que, conforme destacado por Piaget (1973) é um dos pressupostos da ação cooperativa. O wiki mostra-se um ambiente muito propício para este tipo de ação, uma vez que apresenta uma estrutura homogênea em termos do que cada participante pode fazer. Além disso, facilita a interação e a comunicação entre integrantes de um grupo, permitindo que cada um reveja posturas e pontos de vista ao atuar com colegas. A facilidade de construir páginas mantém a atenção de quem utiliza o ambiente no conteúdo sendo criado e não a desloca para a linguagem envolvida na formatação deste conteúdo.

No entanto, o uso efetivo da ferramenta TWiki (implementação de domínio público de um wiki), tanto no PPGIE como na Escola Técnica da UFRGS, evidenciou alguns problemas operacionais e fez com que novos questionamentos fossem levantados sobre as dificuldades da utilização efetiva de wikis no processo de aprendizagem. Perceberam-se carências que dificultam a sua utilização como um ambiente colaborativo. Esta questão também é colocada por diversos autores arrolados na seção 
dois que, a partir de suas experiências com wiki em sala de aula, propõem modificações para adaptar o modelo e torná-lo mais propício para a utilização no ensino.

O presente artigo relaciona, inicialmente, uma coleção de pesquisas já realizadas neste sentido. A seguir, descreve as dificuldades percebidas no uso da ferramenta na UFRGS, levando em consideração o Modelo de Colaboração 3C de Fuks (2004). Finalmente, propõe modificações que incrementariam o processo colaborativo propiciado pelo ambiente, com vistas à aprendizagem.

\section{A utilização do wiki no processo de aprendizagem colaborativa}

Vários são os autores que discutem a utilização de wikis para a aprendizagem colaborativa. Os autores aqui citados não colocam em cheque a utilidade da ferramenta, mas apresentam a necessidade de modificações para que ela se torne mais útil no contexto educacional.

Wang e Turner (2004) identificaram problemas associados com o paradigma clássico do wiki para utilização em sala de aula. Este é um estudo muito interessante, pois apresenta como problemáticas para utilização em sala de aula exatamente aquelas características que fazem parte da definição do que é um wiki: dinamismo, publicidade e construção simultânea dos hipertextos. Os autores consideram que nem todo conteúdo deve ser modificável, que nem todo conteúdo deve ser público, que a questão da atualização simultânea não pode ser permitida e que, em determinado momento, o material produzido deve parar de evoluir.

Honneger (2005) constata a disseminação dos wikis nas escolas dos países de língua germânica, apresentando três razões para este fato:

- simplicidade de uso - linguagem de marcação extremamente fácil de ser aprendida;

- liberdade de acesso;

- inexistência de estruturas restritivas - não há restrições de workflow.

O mesmo artigo, por outro lado, apresenta as dificuldades para a utilização dos wikis na escola:

- falta de ferramentas wikis nas instituições de ensino;

- falta de capacitação tecnológica para gerenciar o serviço;

- falta de editores WYSIWYG nos wikis;

- cultura do ensino colaborativo;

- falta de wikis em alemão, como intensificador de todos os problemas anteriores.

Hampel, Selke e Vitt (2005) deixam claro que, no processo de construção de conhecimento, é fundamental que cada aluno esteja ciente das atividades desenvolvidas pelos colegas. Embora afirmem que o wiki automaticamente apresenta esta informação pelo próprio processo de construção do hipertexto, também apontam que isto nem sempre é nítido para o usuário. Deixam clara a falta de uma forma de comunicação mais explícita, necessária para discutir problemas e negociar soluções.

Elrufae e Turner (2005) propõem algumas características que os wikis devem apresentar para serem utilizados em cursos superiores:

- instrutores devem ter permissão para restringir a possibilidade de modificação de certas páginas;

- estudantes devem ter permissão para tornar páginas privadas durante um período; 
- cada classe deve ter o seu próprio conjunto de páginas wiki e a administração destas páginas deve estar de acordo com as necessidades do instrutor.

Além dessas características, os autores apontam que um novo conjunto de tipos de usuários deve existir nos wikis. Tais tipos de usuários devem se aproximar daqueles existentes em ambientes de aprendizagem (instrutor, aluno, administrador, professor responsável). Também Lund e Smørdal (2006) mostram que a organização dos usuários no wiki utilizado por eles (WikiMedia) não corresponde àquela que se faz em Ambientes Virtuais de Aprendizagem.

Para Notari (2006), o wiki é uma ferramenta poderosa para ambientes de aprendizagem construtivista, uma vez que facilita a colaboração entre os participantes. Porém apresenta, no seu estudo, a dificuldade de fazer com que os estudantes se envolvam no trabalho dos colegas que constituem a sua comunidade de aprendizagem. Cita a necessidade de se criar uma cultura de comunicação e comentário, além da importância da negociação entre os participantes. Também reafirma o papel do professor como proponente de atividades para um determinado intervalo de tempo e como alguém que corrige aquilo que deve ser corrigido.

Por último, Chong e Yamamoto (2006) apresentam a falta de mecanismos de coordenação e comunicação nos wikis. Este último trabalho é aqui citado porque parece resumir a preocupação de todos os outros autores, já que todos eles, de alguma forma, percebem o wiki como um ambiente que permite a realização de tarefas em conjunto, mas identificam problemas. Tais problemas não se relacionam exatamente com a realização da tarefa, ou seja não dizem respeito ao local de cooperação. Relacionam-se, de fato, com aquilo que permite que a cooperação ocorra e que seja realmente proveitosa. Considerando o Modelo de Colaboração 3C (Fuks et alli, 2004), que aponta que em um ambiente colaborativo

"as tarefas se originam dos compromissos e conhecimentos negociados durante a comunicação e são realizadas durante a cooperação e a coordenação atua para garantir que as tarefas sejam executadas da maneira correta, no tempo certo e com os recursos necessários",

os problemas apontados podem ser enquadrados como problemas de coordenação e comunicação. Se os autores que sugerem modificações no ambiente wiki forem lidos à luz deste modelo, poder-se-á notar que todas as sugestões se enquadram em questões de coordenação e comunicação.

\section{A experiência na UFRGS}

Durante o ano de 2005, o PPGIE e a Escola Técnica da UFRGS trabalharam, de forma sistemática, com a ferramenta TWiki em atividades didáticas. TWiki é uma implementação de domínio público do modelo wiki. Foram instalados dois servidores, um para o PPGIE e outro para a Escola Técnica. As questões levantadas nesta seção são resultado da experiência do autor do presente artigo como administrador do ambiente nos dois locais, como professor que o utilizou com seus alunos da Escola Técnica e como aluno que teve de utilizá-lo para construir páginas em disciplina do PPGIE.

Embora o ambiente seja extremamente útil para o trabalho colaborativo, uma vez que tem uma interface muito amigável para que os participantes de grupos de aprendizagem construam textos em conjunto, existe uma deficiência no que diz respeito às atividades de coordenação e comunicação. Isto se explica porque a idéia original do wiki é, justamente, permitir uma construção absolutamente igualitária e dinâmica de hipertextos. 
É certo que na aprendizagem colaborativa são necessários mecanismos que permitam ao professor coordenar os processos de aprendizagem. Não só aos professores, pois também os alunos devem se articular para que suas atividades sejam desenvolvidas de maneira coordenada. Os próprios alunos, que constituem grupos de aprendizagem colaborativa, estabelecem de alguma forma uma coordenação de suas atividades. Também é importante que os participantes percebam a interação como elemento fundamental na construção do conhecimento. Esta percepção conduz a uma participação mais efetiva e se dá com a utilização de mecanismos explícitos. De acordo com Fuks (2004), atividades cujas tarefas são altamente interdependentes não são coordenadas apenas pelo protocolo social. Assim, a utilização das próprias páginas construídas pelos participantes dos grupos para atividades de coordenação e comunicação parece não ser o meio mais indicado para explicitar estas atividades e permitir uma eficiente colaboração.

\subsection{Coordenação}

Para garantir o cumprimento de atividades é necessário que elas sejam coordenadas. Quando os autores citados na seção dois colocam problemas como o controle de acesso (quem pode ver ou editar e quando pode ver ou editar), a atualização simultânea de páginas, os papéis dos usuários, a distribuição de tarefas e o controle do tempo, estão indicando problemas relacionados com a coordenação das atividades no ambiente colaborativo. Problemas desta ordem também puderam ser constatados durante o uso do wiki na UFRGS.

Cabe notar que o TWiki apresenta soluções para a maior parte dos problemas, mas as soluções não estão acessíveis para um professor responsável por uma turma e que não seja administrador do sistema. Isto porque as soluções freqüentemente são obtidas com o que se pode chamar de configuração de baixo nível que envolve configurações realizadas através de comandos textuais. Além do mais, estas configurações normalmente são realizadas no próprio hipertexto, tornando confuso o processo, uma vez que ele não está dissociado do local onde ocorre a cooperação.

O controle de acesso à página é factível no TWiki, uma vez que ele permite que se definam, para cada página, os usuários que podem modificá-la ou visualizá-la. Todavia, existem as seguintes dificuldades:

- o controle do acesso é feito por diretivas textuais dentro da própria edição da página;

- o controle de acesso depende da criação de usuários e da definição de grupos, feita apenas por administradores;

- as autorizações não se replicam para novas páginas definidas, o que torna cansativo o processo de definir as autorizações a cada nova página criada.

Surge um dilema: ou se permite que o professor crie grupos e se dá a ele poder de administrador, ou se mantém a segurança do sistema e o professor perde a independência para realizar uma atividade trivial de coordenação como a criação de grupos. Além disso, mesmo que se permita ao professor realizar esta atividade, não há uma interface adequada se considerarmos que o professor não é um especialista em informática. Isto fica ainda mais crítico quando se considera que os próprios alunos poderiam definir grupos internos para divisão de tarefas.

O problema relatado acima já aponta a necessidade de que os papéis classicamente existentes nos wikis - administrador, usuário registrado e visitante - sejam modificados. Certamente, o professor não é administrador, mas tem um papel 
diferenciado; e o aluno de um curso não desempenha o mesmo papel nas páginas pertencentes ao seu grupo e nas páginas utilizadas por outros grupos de trabalho.

$\mathrm{O}$ acesso simultâneo a uma página foi tratado no TWiki, que permite vários acessos concorrentes e apresenta uma consolidação do que foi feito simultaneamente em uma página. No entanto, não há possibilidade de os participantes decidirem se esta é a melhor solução, ou se a página deveria ser utilizada por um participante de cada vez. Aqui fica claro o primeiro problema: a falta de flexibilidade no tratamento do acesso concorrente. Além disso, levando em consideração a opção de permitir a edição simultânea, uma página editada por mais de um participante deve sofrer a análise destes mesmos participantes para que possam chegar a uma conclusão a respeito do conteúdo final. Mesmo que a conclusão seja de que não há acordo. Eis que, se ambos estão editando, ambos estão conectados e, portanto, podem negociar de forma síncrona, através de um chat, os conflitos existentes, tornando o trabalho muito mais colaborativo.

Coordenar as ações é, também, estabelecer objetivos, prazos e ordem de tarefas. O ambiente não apresenta um mecanismo explícito para este fim. Embora o professor possa passar estas informações através de uma página do próprio wiki, a utilização de mecanismos exclusivos e integrados ao ambiente permitiria que os participantes distinguissem melhor o que é informação de controle e o que é efetivamente produção. Apesar de isto parecer um contra-senso na utilização de uma ferramenta que se baseia na eqüidade entre os participantes, não se deve considerar que apenas os professores fariam uso deste tipo de mecanismo. Os próprios alunos têm de estabelecer tarefas, objetivos, prazos e um fluxo de trabalho, na medida em que interagem. O registro destes compromissos estabelecidos entre os participantes do grupo de aprendizagem em uma estrutura diferente do hipertexto é importante para que, no decorrer do trabalho, haja formas fáceis de recuperá-los e, portanto, realizar uma avaliação mais criteriosa se as atividades cumpriram com seus objetivos, nos prazos estipulados. A avaliação de que o trabalho está caminhando corretamente dá segurança ao grupo de alunos.

A percepção daquilo que o outro está realizando é muito importante no trabalho colaborativo (Gutwin, Stark \& Greenberg, 1995). Mesmo que o TWiki apresente um histórico que permite aos usuários verificar o que foi modificado, este histórico não é orientado para o grupo. O que se necessita é relacionar os objetivos propostos e acordados, as tarefas distribuídas e os resultados alcançados. Este tipo de histórico é certamente mais útil para a atividade de coordenação. Ao entrar no ambiente, o usuário deveria ser notificado das discussões em andamento, das avaliações feitas sobre os seus textos, das modificações realizadas por seu grupo de trabalho e das novas questões propostas. Por isso, todas estas informações não devem estar guardadas na página que contém a produção. Devem estar relacionadas com a página, mas não devem estar codificadas nela, pois constituem informações de controle que devem disparar ações autônomas do sistema.

\subsection{Comunicação}

A despeito de o wiki ser uma ambiente que propicia a comunicação, discute-se aqui, como deve ocorrer a comunicação entre os participantes de um grupo de aprendizagem colaborativa. Durante a construção do hipertexto há uma questão de coordenação e comunicação não tratada pelo modelo wiki: a negociação entre os participantes para se obter o resultado final ou mesmo para se avançar em direção ao resultado pretendido. Embora o ambiente permita que sejam modificados os textos existentes ou acrescentados novos textos, não há um mecanismo explícito para que os 
participantes discutam a pertinência de uma idéia ou decidam sobre possibilidades diferentes. Evidentemente que o próprio ambiente poderia ser utilizado para as discussões e para a tomada de decisão. Inclusive, a implementação WikiMedia, utilizada para a Wikipedia, apresenta lugar de discussão. Mas, mesmo nesta ferramenta, o lugar de discussão é uma página de hipertexto. Ferramentas nos moldes de fóruns e chats são mais adequadas para a negociação com vistas à tomada de decisão, uma vez que apresentam um formato mais parecido com aquele executado em um grupo de trabalho presencial. Além disso, um local separado (um mecanismo explícito) permite que os participantes associem uma função diferente para aquele local e identifiquem o que está ocorrendo. Há necessidade de que todos percebam, na construção das páginas, momentos de decisão explicitamente indicados por um local de discussão. Isto ajuda a criar a cultura da colaboração. Assim, um wiki com o objetivo de fornecer um ambiente colaborativo para a aprendizagem necessita que, para cada página ou conjunto de páginas, sejam associadas ferramentas como fóruns, chats e, até mesmo, mecanismos de votação para o grupo resolver pendências. Alunos pesquisados por Raitman, Augar e Zhou (2005) criticaram justamente a falta de discussões quando da utilização do ambiente.

Deve ser possível para a coordenação decidir que ferramentas de comunicação estarão disponíveis e como serão utilizadas. Também é importante notar que estes mecanismos de comunicação são importantes para que o professor se comunique com os alunos e coordene o processo de aprendizagem. A dificuldade de comunicação afeta diretamente a coordenação.

\section{Conclusão}

Embora seja possível utilizar o wiki como um ambiente para aprendizagem colaborativa, existem lacunas que devem ser preenchidas a fim de que o ambiente atenda às peculiaridades deste tipo de aprendizagem. Estas lacunas estão relacionadas com as atividades de coordenação e comunicação definidas no Modelo de Colaboração 3C. Assim, para que o wiki torne-se mais robusto neste contexto é necessário implementar mecanismos explícitos de coordenação e comunicação. Estes mecanismos explícitos devem ser amigáveis, pois não serão utilizados por técnicos em informática, mas por educadores. Por serem mecanismos explícitos, não podem estar inseridos nos hipertextos produzidos, mas devem estar relacionados com cada uma das páginas, pois são eles que permitirão que o hipertexto gerado realmente seja resultante de um trabalho colaborativo.

Resta o desafio de incluir estes mecanismos sem deixar que isto torne o wiki mais uma ferramenta repleta de restrições. Para isso, os mecanismos de coordenação e comunicação deverão ser os facilitadores da cooperação e não os seus inibidores.

\section{Bibliografia}

CHONG, S. T. \& Yamamoto, M. Collaborative learning using wiki and flexnetdiscuss: a pilot study. In: Proceedings of the 5th IASTED international conference on Web-based education WBE'06. 2006, Puerto Vallarta, Mexico. Anais: ACTA Press, p.150-154.

ELRUFAIE, E.\& TURNER, D.A. A wiki paradigm for use in IT courses. Proceedings of the International Conference on Information Technology: Coding and Computing. 2005. Anais: vol. 2, p. 770-771. 
FUKS, H.; GEROSA, M. A.; RAPOSO, A. B. \& LUCENA, C. J. P. O modelo de colaboração $3 \mathrm{C}$ no ambiente aulanet. Infomática na Educação: Teoria e Prática, Porto Alegre, Vol 7, No 1, p. 25-48, 2004.

HAMPEL, T.; SELKE, H. \& VITT, S. Deployment of simple user-centered collaborative technologies in educational institutions - experiences and requirements. In: Proceedings of the 14th IEEE International Workshops on Enabling Technologies: Infrastructure for Collaborative Enterprise. 2005. Anais: p. 207-212.

HONEGGER, B. D. Wikis: a rapidly growing phenomenon in the german-speaking school community. In: Proceedings of the 2005 international symposium on Wikis. 2006, San Diego, Califórnia. Anais: ACME Press, p.113-116.

LUND, A. \& SMORDAL, O. Is there a space for the teacher in a wiki? In: Proceedings of the 2006 international symposium on Wikis. 2006, Odense, Denmark. Anais: ACME Press, p.37-45.

NOTARI, M. How to use a wiki in education: 'wiki based effective constructive learning'. In: Proceedings of the 2006 international symposium on Wikis. 2006, Odense, Denmark. Anais: ACME Press, p.131-132.

PIAGET, Jean. Estudos Sociológicos. Rio de Janeiro: Forense, 1973.

RAITMAN, R.; AUGAR, N. \& ZHOU, W. Employing wikis for online collaboration in the e-learning environment: case study. In: Proceedings of the Third International Conference on Information Technology and Applications. 2005. Anais: vol.2, p.142-146.

TAROUCO, L. et alli. Twiki, uma ferramenta de co-autoria livre. RENOTE : revista novas tecnologias na educação, Porto Alegre, nov. 2005. Disponível em: < http://www.cinted.ufrgs.br/renote/nov2005/index.html> . Acesso em: $10 \mathrm{dez}$. 2006.

WANG, C. \& TURNER, D. Extending the wiki paradigm for use in the classroom. In: Proceedings of the International Conference on Information Technology: Coding and Computing. 2004. Anais: vol.1, p.255-259. 